\title{
BMJ Open Minocycline for the treatment of mental health and neurological conditions: study protocol of a systematic review and meta-analysis
}

Chiara C Bortolasci (D , ${ }^{1}$ Wolfgang Marx, ${ }^{1}$ Adam J Walker, ${ }^{1}$ Kyoko Hasebe, ${ }^{1,2}$ Bianca E Kavanagh, ${ }^{1}$ Margaret J Morris, ${ }^{2}$ Mohammadreza Mohebbi, ${ }^{3}$ Alyna Turner, ${ }^{1,4,5}$ Laura Gray, ${ }^{1}$ Lesley Berk, ${ }^{1,6}$ Ken Walder, ${ }^{1}$ Michael Berk, ${ }^{1}$ Olivia M Dean ${ }^{1}$

To cite: Bortolasci CC, Marx W, Walker AJ, et al. Minocycline for the treatment of mental health and neurological conditions: study protocol of a systematic review and meta-analysis. BMJ Open 2020;10:e035080. doi:10.1136/ bmjopen-2019-035080

- Prepublication history and additional material for this paper are available online. To view these files, please visit the journal online (http://dx.doi. org/10.1136/bmjopen-2019035080).

Received 17 October 2019 Revised 12 February 2020 Accepted 21 February 2020

Check for updates

(C) Author(s) (or their employer(s)) 2020. Re-use permitted under CC BY-NC. No commercial re-use. See rights and permissions. Published by BMJ.

For numbered affiliations see end of article.

Correspondence to Dr Chiara C Bortolasci; bchiara@deakin.edu.au

\section{ABSTRACT}

Introduction Due to the anti-inflammatory, antioxidant and anti-apoptotic properties of minocycline, clinical trials have evaluated the potential of this drug to treat several psychiatric and neurological disorders, including major depressive disorder, schizophrenia, bipolar disorder, stroke and amyotrophic lateral sclerosis. This protocol proposes a systematic review (and potential meta-analysis) that aims to identify and critically evaluate randomised controlled trials of minocycline for treating psychiatric and neurological disorders.

Methods and analysis PubMed, Embase, Cochrane Central Register of Controlled Clinical Trials, PsycINF0 and Cumulative Index to Nursing and Allied Health Literature (CINAHL) will be used to identify randomised controlled trials that used minocycline to treat psychiatric and neurological disorders. Double-blind, randomised, controlled, clinical trials of participants aged 18 years or older and written in English will be included in the review. Data will be extracted by two independent reviewers. Preferred Reporting Items for Systematic Reviews and Meta-Analyses guidelines will be followed and the Cochrane Collaboration's 'Risk of Bias' tool will be used to assess the risk of bias in all studies included in the systematic review. The Grading of Recommendations, Assessment, Development and Evaluation system will be used to access the overall quality of the level of evidence of the studies. If sufficient evidence is identified, a meta-analysis will be conducted using the standardised mean difference approach and reported with $95 \% \mathrm{Cls}$. Heterogeneity of evidence will be evaluated using the $\mathrm{I}^{2}$ model.

Ethics and dissemination This systematic review will evaluate only published data; therefore, ethical approval is not required. The systematic review will be published in a peer-reviewed journal and presented at relevant research conferences.

Trial registration number CRD42020153292.

\section{INTRODUCTION}

Minocycline (7-dimethylamino-6-demethyl-6deoxytetracycline) is a second-generation semi-synthetic tetracycline antibiotic. It is used
Strengths and limitations of this study

- This systematic review will update and extend the evidence regarding the effects of minocycline in psychiatric and neurological disorders; this is the first review to present these two fields together.

- Data will be extracted by two independent reviewers.

- Rigorous screening processes and assessment of included studies will allow only for peer-reviewed publications.

- A potential limitation of this systematic review may be the lack of studies in some of the conditions, making it unfeasible to perform a meta-analysis.

to treat several bacterial infections as well as severe acne and rheumatoid arthritis. ${ }^{1}$ Minocycline is a highly lipophilic drug and can cross the blood-brain barrier. ${ }^{2}$ It is well tolerated in humans with either acute or chronic use. ${ }^{3}$

Minocycline has been proposed as a novel intervention for psychiatric and neurological disorders due to its anti-inflammatory, antioxidant and anti-apoptotic properties, in addition to its ability to promote neurogenesis. ${ }^{4-6}$ Several neuropsychiatric and neurologic disorders have been associated with neuroinflammation. $^{7-12}$ Minocycline is known to have inhibitory effects on microglial activation. ${ }^{4}$ This inhibition aids to attenuate neuroinflammation and may be beneficial in preventing cognitive and behavioural impairments. In addition, the antioxidant properties of minocycline, including freeradical scavenging activity and inhibition of lipid peroxidation, have been described in several studies. ${ }^{1314}$ Minocycline also presents cytoprotective properties by reducing apoptosis. This can be explained at least in part by its effect on the expression of the antiapoptotic B-cell lymphoma protein-2 (Bcl-2) 
and antagonising pro-apoptotic proteins such as Bax, Bak and Bid. ${ }^{1516}$ This, in turn, suppresses the activation of caspases 1 and 3 and the release of apoptotic factors such as cytochrome c from mitochondria, reducing cell death. ${ }^{15}{ }^{16}$ Minocycline additionally enhances neurogenesis in several preclinical models. ${ }^{1718}$

These aforementioned properties led to several studies designed to evaluate the potential of minocycline to treat several psychiatric and neurological disorders, including major depressive disorder (MDD), schizophrenia, bipolar disorder, stroke and amyotrophic lateral sclerosis (ALS). Regarding the current use of minocycline in psychiatry, there have been recent systematic reviews from Zheng et $a l^{19}$ reporting that adjunctive minocycline treatment improved total symptoms in schizophrenia patients, but failed to demonstrate primary efficacy for bipolar depression and MDD. With respect to MDD, the findings of Zheng $e t$ al are in contrast to the work of Rosenblat and McIntyre which showed a positive effect of minocycline on depressive symptoms. ${ }^{20}$ This can be explained by the fact that Zheng et al only included studies with minocycline as monotherapy and Rosenblat and McIntyre included studies where minocycline was an adjunctive treatment. Similarly, secondary outcomes were not explored by Zheng and colleagues. On the other hand Xiang et $a l^{21}$ and Solmi $e t a l^{22}$ also demonstrated a potential positive effect of minocycline in schizophrenia.

In neurology, Malhotra $e t a t^{23}$ reviewed the effects of minocycline in acute ischaemic stroke (AIS) and acute intracerebral haemorrhage showing efficacy especially in the AIS group. The potential of minocycline in Parkinson's disease, Huntington's disease and ALS has been previously discussed in the literature; however to the best of our knowledge, no systematic review has been conducted to evaluate the effects of minocycline in these conditions.

In animal models, some deleterious effects of minocycline have been reported in the literature. Diguet $e t$ al suggest that minocycline may have deleterious effects in Parkinson's models according to the mode of administration and dose. ${ }^{24}$ Vogt et al showed no antidepressant effects at doses from 20 to $40 \mathrm{mg} / \mathrm{kg}$ in a C57BL $/ 6$ mouse. ${ }^{25}$ Minocycline ameliorated brain injury in developing rats, but worsened the injury in the developing C57BL/ 6 mouse. ${ }^{26}$ Pro-apoptotic properties of minocycline were demonstrated in developing mouse brain, raising concerns of its use in children. ${ }^{27}$

There is clear justification to explore the potential use of minocycline across psychiatric and neurological disorders that share dysregulation in inflammation, oxidative pathways, apoptosis and neurogenesis. There are significant overlaps in pathophysiology between many psychiatric and neurological conditions. Minocycline potentially addresses these common factors, such as inflammation and oxidative stress.

Since the publication of earlier reviews ${ }^{628}$ more data are available that may enhance the understanding of the clinical utility of minocycline across neuropsychiatry.
More studies allow for exploration of secondary outcomes and subgroups, especially in disorders with high clinical heterogeneity such as depression. As mentioned earlier, there is no systematic evaluation of the use of minocycline in neurology; thus given its use in this field, bringing together the current evidence would be both timely and valuable. Therefore, there is value in exploring the evidence regarding the use of minocycline across fields to better understand its potential clinical usefulness.

\section{OBJECTIVES}

This systematic review aims to identify and critically evaluate randomised controlled trials of minocycline for treating psychiatric and neurological disorders. A comprehensive understanding of the current level of evidence in the literature will help clarify the clinical utility of minocycline in each disease and inform future research.

\section{METHODS AND ANALYSIS}

This systematic review will be conducted in accordance with the Preferred Reporting Items for Systematic Reviews and Meta-Analyses (PRISMA) guidelines. ${ }^{29}$ The Preferred Reporting Items for Systematic review and Meta-Analysis Protocols (PRISMA-P) checklist is attached as online supplementary file 1 . It will include randomised controlled trials of minocycline of any size and duration in adult populations ( $\geq 18$ years). The design of the studies can be placebo or active-controlled, parallel group or cluster design. Studies which have included more than one pharmacological intervention will also be included, with the data being pooled when possible. Only studies published in English will be included. Additional information will be requested from the study authors if insufficient details are provided in the manuscript. All studies conducted to the present will be included.

The primary outcome of this systematic review will be improvement in core psychiatric or neurological symptoms after treatment with minocycline. Secondary outcomes include assessing the effects of minocycline treatment on quality of life, functioning, cognition, anxiety, disability, survival time and time to relapse. Adverse drug reactions and discontinuation rates will also be evaluated.

\section{Search strategy}

The PICO (Patient/Problem/Population, Intervention, Comparison/Control, Outcome) framework was used to develop the search strategy for this review. PubMed, Embase via embase.com, Cochrane Central Register of Controlled Clinical Trials via cochranelibrary.com, PsycINFO via EBSCO and CINAHL Complete via EBSCO will be used to search for the relevant literature up to March 2020. The appropriate index terms for each database will be used. The online supplementary file 2 shows the full search strategy for PubMed and will be adapted to fit with other databases. The references cited in the 
included publications will be searched for additional studies. Studies published since journal inception to the date the searches are run will be included. The searches will be re-run prior to the final analyses and any further studies identified will be retrieved for inclusion.

The studies extracted from the search will be checked for eligibility based on a selection of criteria. First, the titles and abstracts will be assessed to determine if the study is a randomised controlled trial, investigating an intervention with minocycline and has been conducted on participants with a psychiatric or neurologic disease. Subsequently, the full-text papers will be reviewed. A second reviewer will evaluate all articles at each stage of screening. If there is disagreement regarding eligibility, a third independent reviewer will determine the conclusive decision.

\section{Data management}

An online reference management database, Covidence, ${ }^{30}$ will be used to manage the data. Covidence allows abstracts and full text screening, handling of duplicate data, assessment of the risk of bias and extraction of the study outcomes based on inclusion and exclusion criteria.

Data will be extracted by two reviewers and it will include: (a) study identification details, (b) study design, (c) population characteristics, (d) intervention details, (e) outcome measurements, (f) information for assessment of the risk of bias. Authors will be contacted where substantial outcomes of interest are not reported and/or clarification about study design is required.

\section{Assessment of methodological quality}

The Cochrane Collaboration's 'Risk of Bias' tool will be used to assess the risk of bias in all studies included in this review. ${ }^{31}$ This tool covers six domains of bias: selection bias, performance bias, detection bias, attrition bias, reporting bias, and other bias and scores the randomised trials as low, high or unknown risk of bias. The overall quality of the level of evidence of the studies will be measured with the Grading of Recommendations, Assessment, Development and Evaluation system. ${ }^{32}$

\section{Data synthesis and statistical analysis}

A narrative synthesis of the findings will be reported, including population characteristics, type of outcome and intervention details.

If sufficient data are available, a meta-analysis will be performed for each diagnostic category. ${ }^{30-33}$ For continuous data, mean differences or standardised mean differences with 95\% CIs will be calculated. Dichotomous data will be analysed using risk ratios with $95 \%$ CIs. Mean differences will be used when the treatment outcome has been measured by the same scale. Standardised mean differences will be used when the treatment outcome has been measured by different scales in different studies. Sample size, SD and $\mathrm{p}$ values will be reported. Significance will be set at $\alpha \leq 0.05$.
To assess the statistical heterogeneity of evidence, $\mathrm{I}^{2}$ will be used in accordance with the suggestions from the Cochrane Handbook for Systematic Reviews of Interventions 5.1. ${ }^{34}$ Studies will be scored as low $\left(\mathrm{I}^{2}\right.$ value of $\left.25 \%\right)$, moderate $\left(\mathrm{I}^{2}\right.$ value of $\left.50 \%\right)$ or high $\left(\mathrm{I}^{2}\right.$ value of $\left.75 \%\right)$.

Sensitivity analysis will be performed to evaluate the robustness of the meta-analysis outcome.

\section{Subgroup analysis}

The following subgroup analyses will be performed if sufficient data are available:

- Trial treatment duration.

- Effect of treatment at different doses.

- Patient demographics (age, gender and illness severity).

- Biomarkers of inflammation and oxidative stress.

\section{Presentation and reporting of results}

This review will follow the PRISMA-P guidelines ${ }^{35}$ for reporting data. A PRISMA-P flow chart will describe study selection and numbers excluded at each stage of the process (and the appropriate reasons for exclusion).

\section{Ethics and dissemination}

Ethical approval is not required since this review will only include published data which already received ethical approval prior to publication. This review has been registered on PROSPERO. The results of this systematic review will be published in a peer-reviewed journal and presented at relevant scientific conferences.

\section{No patient and public involvement}

This research was done without patient involvement. Patients were not invited to comment on the study design and were not consulted to develop patient relevant outcomes or interpret the results. Patients were not invited to contribute to the writing or editing of this document for readability or accuracy.

\section{Author affiliations}

${ }^{1}$ The Institute for Mental and Physical Health and Clinical Translation, Deakin University, Barwon Health, Geelong, Victoria, Australia

${ }^{2}$ School of Medical Sciences, UNSW Sydney, Sydney, New South Wales, Australia

${ }^{3}$ Biostatistics Unit, Faculty of Health, Deakin University, Melbourne, Victoria, Australia ${ }^{4}$ Department of Psychiatry, Royal Melbourne Hospital, University of Melbourne,

Parkville, Victoria, Australia

${ }^{5}$ School of Medicine and Public Health, Faculty of Health and Medicine, University of Newcastle \& Hunter Medical Research Institute, Callaghan, New South Wales, Australia

${ }^{6}$ Melbourne School of Population and Global Health, University of Melbourne, Carlton, Victoria, Australia

Acknowledgements The authors wish to thank Blair Kelly, Deakin University, for advice on the search strategy and library support.

Contributors CB conceptualised and designed the research questions and search strategy, prepared the manuscript draft, edited and approved the final version of the manuscript. WM designed the research questions and search strategy, edited the manuscript and approved the final version. AW, KH, BK, MM, MJM, AT, LG, LB, $\mathrm{KW}$ and $\mathrm{MB}$ revised the search strategy, edited and approved the manuscript. OMD conceptualised the research question and search strategy, edited the manuscript and approved the final version. 
Funding The authors have not declared a specific grant for this research from any funding agency in the public, commercial or not-for-profit sectors.

Competing interests OMD has received grant support from the Brain and Behavior Foundation, Simons Autism Foundation, Stanley Medical Research Institute, Deakin University, Lilly, National Health and Medical Research Council (NHMRC) and Australasian Society for Bipolar and Depressive Disorders/Servier. MJM has received grant support from NHMRC.

Patient and public involvement Patients and/or the public were not involved in the design, or conduct, or reporting or dissemination plans of this research.

Patient consent for publication Not required.

Provenance and peer review Not commissioned; externally peer reviewed.

Open access This is an open access article distributed in accordance with the Creative Commons Attribution Non Commercial (CC BY-NC 4.0) license, which permits others to distribute, remix, adapt, build upon this work non-commercially, and license their derivative works on different terms, provided the original work is properly cited, appropriate credit is given, any changes made indicated, and the use is non-commercial. See: http://creativecommons.org/licenses/by-nc/4.0/.

ORCID iD

Chiara C Bortolasci http://orcid.org/0000-0002-0794-6363

\section{REFERENCES}

1 Nagarakanti S, Bishburg E. Is minocycline an antiviral agent? A review of current literature. Basic Clin Pharmacol Toxicol 2016;118:4-8.

2 Chopra I, Roberts M. Tetracycline antibiotics: mode of action, applications, molecular biology, and epidemiology of bacterial resistance. Microbiol Mol Biol Rev 2001;65:232-60.

3 Garner SE, Eady A, Bennett C, et al. Minocycline for acne vulgaris: efficacy and safety. Cochrane Database Syst Rev 2012:Cd002086.

4 Clemens V, Regen F, Le Bret N, et al. Anti-Inflammatory effects of minocycline are mediated by retinoid signaling. BMC Neurosci 2018;19:58.

5 Garrido-Mesa N, Zarzuelo A, Gálvez J. Minocycline: far beyond an antibiotic. Br J Pharmacol 2013;169:337-52.

6 Dean OM, Data-Franco J, Giorlando F, et al. Minocycline: therapeutic potential in psychiatry. CNS Drugs 2012;26:391-401.

7 Data-Franco J, Singh A, Popovic D, et al. Beyond the therapeutic shackles of the monoamines: new mechanisms in bipolar disorder biology. Prog Neuropsychopharmacol Biol Psychiatry 2017;72:73-86.

8 Anderson G, Berk M, Dean O, et al. Role of immune-inflammatory and oxidative and nitrosative stress pathways in the etiology of depression: therapeutic implications. CNS Drugs 2014;28:1-10.

9 Khandaker GM, Cousins L, Deakin J, et al. Inflammation and immunity in schizophrenia: implications for pathophysiology and treatment. Lancet Psychiatry 2015;2:258-70.

10 Spangenberg EE, Green KN. Inflammation in Alzheimer's disease: lessons learned from microglia-depletion models. Brain Behav Immun 2017:61:1-11.

11 Réus GZ, Fries GR, Stertz L, et al. The role of inflammation and microglial activation in the pathophysiology of psychiatric disorders. Neuroscience 2015;300:141-54.

12 Rana A, Musto AE. The role of inflammation in the development of epilepsy. J Neuroinflammation 2018;15:144.

13 Kraus RL, Pasieczny R, Lariosa-Willingham K, et al. Antioxidant properties of minocycline: neuroprotection in an oxidative stress assay and direct radical-scavenging activity. J Neurochem 2005;94:819-27.
14 Soory M. A role for non-antimicrobial actions of tetracyclines in combating oxidative stress in periodontal and metabolic diseases: a literature review. Open Dent J 2008;2:5-12.

15 Zhang $\mathrm{L}$, Huang $\mathrm{P}$, Chen $\mathrm{H}$, et al. The inhibitory effect of minocycline on radiation-induced neuronal apoptosis via AMPK $\alpha 1$ signalingmediated autophagy. Sci Rep 2017;7:16373.

16 Wang J, Wei Q, Wang C-Y, et al. Minocycline up-regulates Bcl2 and protects against cell death in mitochondria. J Biol Chem 2004;279:19948-54.

17 Lu Y, Giri PK, Lei S, et al. Pretreatment with minocycline restores neurogenesis in the subventricular zone and subgranular zone of the hippocampus after ketamine exposure in neonatal rats. Neuroscience 2017;352:144-54.

18 Miao H, Li R, Han C, et al. Minocycline promotes posthemorrhagic neurogenesis via M2 microglia polarization via upregulation of the TrkB/BDNF pathway in rats. J Neurophysiol 2018;120:1307-17.

19 Zheng W, Zhu X-M, Zhang Q-E, et al. Adjunctive minocycline for major mental disorders: a systematic review. J Psychopharmacol 2019;33:1215-26.

20 Rosenblat JD, Mclntyre RS. Efficacy and tolerability of minocycline for depression: a systematic review and meta-analysis of clinical trials. J Affect Disord 2018;227:219-25.

21 Xiang $Y-Q$, Zheng W, Wang S-B, et al. Adjunctive minocycline for schizophrenia: a meta-analysis of randomized controlled trials. Eur Neuropsychopharmacol 2017;27:8-18.

22 Solmi M, Veronese N, Thapa N, et al. Systematic review and metaanalysis of the efficacy and safety of minocycline in schizophrenia. CNS Spectr 2017;22:415-26.

23 Malhotra K, Chang JJ, Khunger A, et al. Minocycline for acute stroke treatment: a systematic review and meta-analysis of randomized clinical trials. J Neurol 2018;265:1871-9.

24 Diguet E, Fernagut P-O, Wei X, et al. Deleterious effects of minocycline in animal models of Parkinson's disease and Huntington's disease. Eur J Neurosci 2004;19:3266-76.

25 Vogt MA, Mallien AS, Pfeiffer N, et al. Minocycline does not evoke anxiolytic and antidepressant-like effects in C57BL/6 mice. Behav Brain Res 2016;301:96-101.

26 Tsuji M, Wilson MA, Lange MS, et al. Minocycline worsens hypoxicischemic brain injury in a neonatal mouse model. Exp Neurol 2004;189:58-65.

27 Inta I, Vogt MA, Vogel AS, et al. Minocycline exacerbates apoptotic neurodegeneration induced by the NMDA receptor antagonist MK801 in the early postnatal mouse brain. Eur Arch Psychiatry Clin Neurosci 2016;266:673-7.

28 Yong VW, Wells J, Giuliani F, et al. The promise of minocycline in neurology. Lancet Neurol 2004;3:744-51.

29 Shamseer L, Moher D, Clarke M, et al. Preferred reporting items for systematic review and meta-analysis protocols (PRISMA-P) 2015: elaboration and explanation. BMJ 2015;350:g7647.

30 Innovation VH. Covidence systematic review software. Available: www.covidence.org

31 Higgins JPT, Altman DG, Gøtzsche PC, et al. The Cochrane collaboration's tool for assessing risk of bias in randomised trials. BMJ 2011;343:d5928.

32 Guyatt GH, Oxman AD, Vist GE, et al. Grade: an emerging consensus on rating quality of evidence and strength of recommendations. $B M J$ 2008;336:924-6.

33 The Nordic Cochrane Centre, The Cochrane Collaboration. Review Manager (RevMan) [Computer program. Copenhagen: The Nordic Cochrane Centre, The Cochrane Collaboration, 2014.

34 Higgins JPT. Cochrane Handbook for systematic reviews of interventions version 5.1.0: the Cochrane collaboration, 2011.

35 Moher D, Shamseer L, Clarke M, et al. Preferred reporting items for systematic review and meta-analysis protocols (PRISMA-P) 2015 statement. Syst Rev 2015;4:1. 